\title{
Assessment of linkage disequilibrium patterns between structural variants and single nucleotide polymorphisms in three commercial chicken populations
}

Johannes Geibel ( $\nabla$ johannes.geibel@uni-goettingen.de )

University of Goettingen

Nora Paulina Praefke

University of Goettingen

Steffen Weigend

Friedrich-Loeffler-Institut

Henner Simianer

University of Goettingen

Christian Reimer

University of Goettingen

\section{Research Article}

Keywords: Chickens, single nucleotide polymorphisms, structural variants, linkage disequilibrium

Posted Date: August 31st, 2021

DOI: https://doi.org/10.21203/rs.3.rs-861830/v1

License: (c) (i) This work is licensed under a Creative Commons Attribution 4.0 International License. Read Full License

Version of Record: A version of this preprint was published at BMC Genomics on March 9th, 2022. See the published version at https://doi.org/10.1186/s12864-022-08418-7. 
1 Assessment of linkage disequilibrium pat-

2 terns between structural variants and sins gle nucleotide polymorphisms in three ${ }_{4}$ commercial chicken populations 5

6 Johannes Geibel ${ }^{12 *}$, Nora Paulina Praefke ${ }^{12}$, Steffen Weigend ${ }^{23}$, Henner Simianer ${ }^{12}$, Christian Reimer ${ }^{12}$

$8{ }^{1}$ Animal Breeding and Genetics Group, Department of Animal Sciences, University of Goettingen, GER-

9 MANY

$10 \quad{ }^{2}$ Center for Integrated Breeding Research, University of Goettingen, GERMANY

$11{ }^{3}$ Institute of Farm Animal Genetics, Friedrich-Loeffler-Institut, GERMANY

*Corresponding author; johannes.geibel@uni-goettingen.de 


\section{Abstract}

\section{Background}

Structural variants (SV) are causative for some prominent phenotypic traits of livestock as different comb types in chickens or color patterns in pigs. Their effects on production traits are also increasingly studied. Nevertheless, accurately calling SV remains challenging. It is therefore of interest, whether close-by single nucleotide polymorphisms (SNPs) are in strong linkage disequilibrium (LD) with SVs and can serve as markers. Literature comes to different conclusions on whether SVs are in LD to SNPs on the same level as SNPs to other SNPs. The present study aimed to generate a precise SV callset from whole-genome short-read sequencing (WGS) data for three commercial chicken populations and to evaluate LD patterns between the called SV and surrounding SNPs.

\section{Results}

The final callset consisted of 12,294,329 bivariate SNPs, 4,301 deletions (DEL), 224 duplications (DUP), 218 inversions (INV) and 117 translocation breakpoints (BND). While average LD between DELs and SNPs was at the same level as between SNPs and SNPs, LD between other SVs and SNPs was strongly reduced (DUP: $40 \%$, INV: $27 \%$, BND: $19 \%$ of between-SNP LD). A main factor for the reduced LD was the presence of local minor allele frequency differences, which accounted for $50 \%$ of the difference between SNP - SNP and DUP - SNP LD. This was potentially accompanied by lower genotyping accuracies for DUP, INV and BND compared with SNPs and DELs. An evaluation of the presence of tag SNPs (SNP in highest LD to the variant of interest) further revealed DELs to be slightly less tagged by WGS SNPs than WGS SNPs by other SNPs. This difference, however, was no longer present when reducing the pool of potential tag SNPs to SNPs located on four different chicken genotyping arrays.

\section{Conclusions}

The results imply that genomic variance due to DELs in the chicken populations studied can be captured by different SNP marker sets as good as variance from WGS SNPs, whereas separate SV calling might be advisable for DUP, INV, and BND effects. 


\section{Keywords}

Chickens; single nucleotide polymorphisms; structural variants; linkage disequilibrium

\section{Background}

A type of genomic variation that affects large regions of the genome is caused by structural variants (SV). SVs can alter the total genome size by deleting (deletions, DEL), duplicating (duplications, DUP) or inserting (insertions, INS) longer stretches of DNA (unbalanced SV). Those SVs are often referred to as copy number variations (CNV). In contrast, inversions (INV) and translocations (TRA) do not affect the length of the genome (balanced SV) [1]. Especially unbalanced SVs are assumed to come with a strong functional impact on the phenotype, e.g. by strong deleterious effects of DELs which can remove complete genes [2] or by DUPs that increase numbers of cis-regulatory elements [2, 3]. SVs and complex combinations of multiple SVs are also known to be causative for some of the most prominent phenotypic breed characteristics of livestock breeds as walnut- and rose comb in chickens [4] or belted color patterns and dominant-white color in pigs [5].

The power for detection of SVs of certain types and sizes, however, is highly technology-dependent in various aspects [1]. During the last two decades, technologies evolved that increased the resolution and accuracy of SV detection at the submicroscopic level. Array-based comparative genomic hybridization (aCGH) allowed the detection of long CNVs >35 kb [2]. The development and increased use of microarrays led to technologies that either detect DELs from characteristics of population-level single nucleotide polymorphism (SNP) genotypes $[6,7]$ or utilized signal intensity information [8]. The increasing availability of short-read sequences during the last decade led to the development of multiple SV detection algorithms which use read depth distributions [1,9] and/or information from split reads and insert size distributions of paired-end reads, potentially combined with local assembly procedures $[1,10-12]$. However, short-read-based methods still come with a variety of limitations due to the short read sizes which highly vary between the algorithms $[1,13]$ and especially a general deficit in calling INS [14]. Therefore, current state-of-the-art methods nowadays utilize the information of PacBio or 
Nanopore long-read sequencing or linked-read technologies as HI-C [15], but the availability of these types of sequencing data is still very limited for the majority of intensively researched livestock species.

Other than for SVs, the use of SNPs has become routine over the last two decades. Therefore, large whole-genome-sequencing (WGS) reference panels $[16,17]$ and collections of individuals, which were genotyped by microarrays and phenotyped in routine breeding programs or during large-scale research projects [18], exist. Given the complexity of SV detection, it is of interest to know which part of the effects of SVs on the phenotype is already captured by potential linkage disequilibrium (LD) between the SV of interest and nearby SNPs. Strong LD would allow for the inclusion of those effects in e.g. genomic prediction without the need for a separate SV analysis.

LD between two variants can be measured using a variety of estimators (reviewed e.g. in [19]), of which the squared correlation of haplotypes $\left(r^{2}\right)$ is probably the most prominent one. It can be interpreted as the amount of information of a variant that is captured by another one. However, its upper limit is defined by the difference in minor allele frequency ( $\triangle \mathrm{MAF}$ ) between the two variants [20]. The overall strength of LD is highly population depended and closely linked to the effective population size [19]. LD thereby shows a characteristic decay pattern of mean LD by distance. However, for many applications as genome-wide association studies (GWAS), the interest is more in the maximum observed LD of a causal variant to a close-by so-called tag SNP, which can capture the effect as a marker genotype. By now, a bunch of studies has addressed the question of linkage between SVs and surrounding SNPS in humans with contrasting results. Generally, common DELs were shown to be in good LD to SNPs by most of the studies $[6,21-24]$, but some found this LD to be weaker than SNP - SNP LD [25, 26]. Literature additionally suggests, that rare DELs are weaker tagged (tag SNP is SNP with highest LD to the variant within a defined distance) than common DELs $[22,27]$ and DUP were in weaker LD to SNPs than DELs $[22,26,28]$. It was additionally shown that the availability of tag SNPs for SVs depends on the SNP panel used (WGS vs. different arrays) [22-24]. A further effect that was found is the location of the SV on the genome. Regions of segmental duplications are known to trigger recurrent SV formation by 
non-allelic homologous recombination and therefore lead to SV hotspots [1,29]. A closer look at those regions by Locke et al. [30] found very few of those CNV to be tagged by surrounding SNPs.

Reduced LD between SNPs and SVs can have diverse reasons. A main factor is the increased possibility of the occurrence of recurrent mutations in regions of low sequence complexity by non-allelic homologous recombination (NAHR)[29]. SVs from recurrent mutational events then show reduced LD to variants from a unique mutational event $[6,30]$. LD between SNPs and SVs may further be decreased by different selectional properties of SNPS and SV [31], MAF differences between SVs and SNPs [20], or ascertainment of SNPs for arrays that excludes regions of high structural complexity due to technical reasons [32]. Additionally, known problems with SV calling accuracy [1] may lead to a high share of false-positive SV calls and therefore on average low LD to more accurately called SNPs.

For livestock, results on SV - SNP LD are very rare, even though a high number of publications targets SV. Based on a GWAS on 26,362 Holstein dairy cattle 50 k genotypes, Xu et al. [33] found a quarter of CNVs that were significantly associated with milk traits not being tagged by adjacent SNPs. The same was observed by Lee et al. [32] who investigated functional and population genetic features of CNV regions in two dairy cattle breeds, also called from a $50 \mathrm{k}$ SNP array. They identified a weak linkage between CNV regions and SNPs, which was slightly stronger between DELs and SNPs than between DUPs and SNPs. Wang et al. [34] included a local LD analysis around CNVs (called from SNP arrays) that were significantly associated with production traits in pigs. Four out of eight significantly associated CNVs overlapped haploblocks of non-significant SNPs, but only one CNV was found $300 \mathrm{~kb}$ downstream of significantly associated SNPs. Note that this, however, may also be an artifact of a much stronger correction for multiple testing in SNPs than in CNV.

In chickens, a variety of studies investigated CNVs on a quantitative basis. The studies either used aCGH [35-39], utilized signal information of SNP arrays [40-45] via PennCNV [8], or read depth information of short-read sequences [46-50]. There were only three studies that also included non-CNV SVs [46, $50,51]$. None of the studies analyzed the LD patterns of the variants. 


\section{Aim of the study}

This is the first study that assessed SV - SNP LD in chickens to investigate the usefulness of SNP markers in capturing SV-based genomic variance. We, therefore, identified SVs from paired-end short-read sequences in three commercial chicken populations (white layers, brown layers, broilers), thoroughly described the SV callset, and assessed the strength of LD between those SVs and SNPs. We also identified major reasons for some existing differences to SNP - SNP LD and evaluated the performance of four available SNP arrays to tag SVs.

\section{Results}

\section{Calling results and description of variants}

For the study, paired-end short-read sequences of 90 chickens from three populations ( 25 commercial white layers, WL; 25 commercial brown layers, BL; 40 commercial broiler chickens, BR) were used. The raw data was first published by Qanbari et al. [52] which contains a more detailed description of the studied populations. SNP genotypes were retrieved from a previous study [53]. SVs were called by a consensus calling approach, which used three paired-end and split-read-based tools, followed by a strict filtering procedure that further utilized read-depth and SNP information. Finally, the remaining SV calls were visually checked by evaluating samplots [54] for each variant, the merged SNP and SV set was phased, and missing genotypes were imputed. The filtering procedure retained 12,294,329 bivariate SNPs, 4,301 DELs, 224 DUPs, 218 INVs, and 117 translocation breakpoints (break ends; BND) on chromosomes 1 - 33. Note that all INS were filtered out due to missing support by at least two variant callers.

Figure $1 \mathrm{~A}$ shows the length distribution of the called SVs. DELs were on average shortest with a median of $443 \mathrm{bp}$ and a maximum of $67,037 \mathrm{bp}$. DUPs (median $=12,285 \mathrm{bp}$; maximum $=778,041 \mathrm{bp}$ ) were larger than DELs and INVs were largest (median $=25,643 \mathrm{bp}$; maximum $=5,795,187 \mathrm{bp}$ ). BNDs only indicate translocation breakpoints and, therefore, do not come with length information. The called SNPs in total accounted for $1.28 \%$ of the autosomal reference genome length, while DELs covered 
140 found in Figure $1 \mathrm{~B}$. We additionally checked how much of the autosomal reference genome is homo-

A

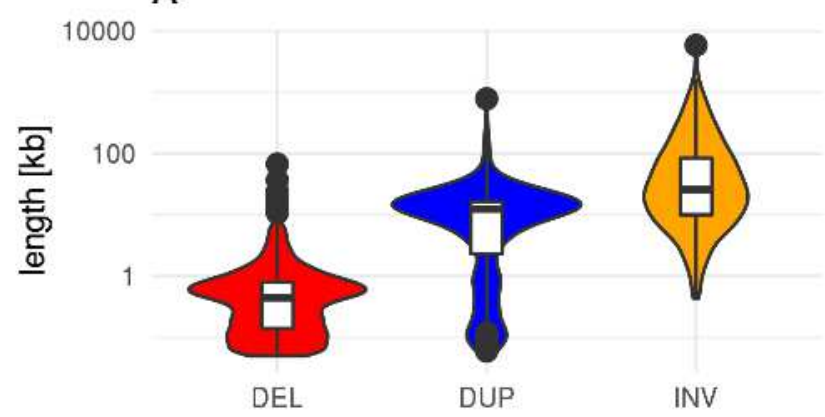

C

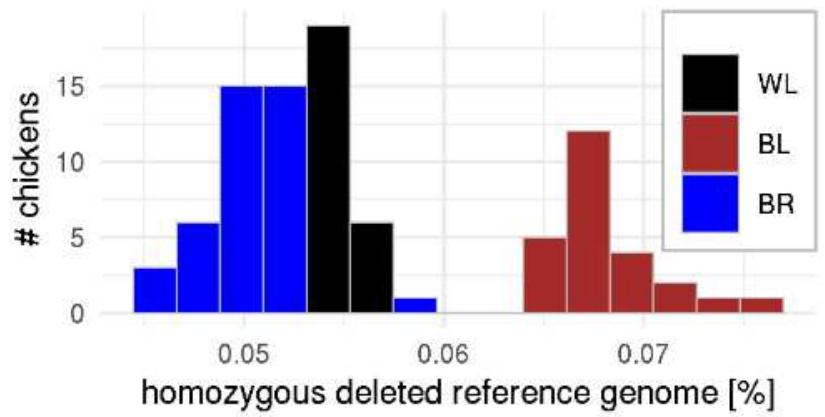

B
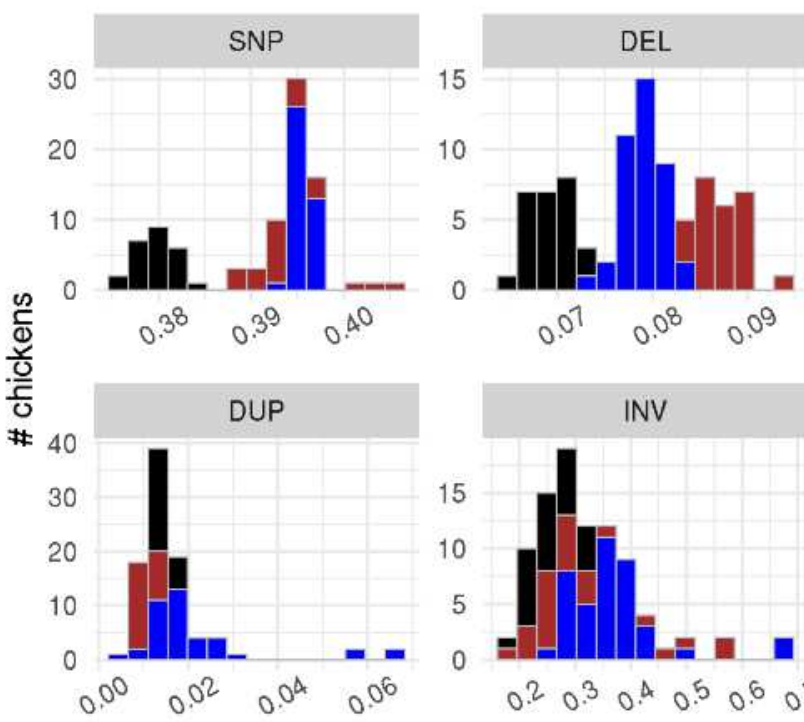

INV

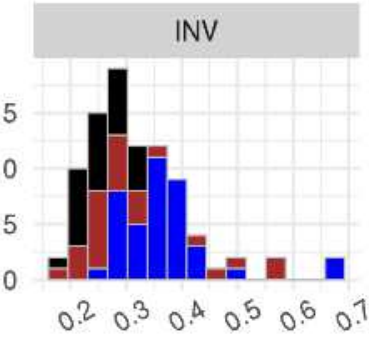

affected reference genome [\%]

WL

$B L$

$B R$

Figure 1: Length distribution of SVs (A), percent of affected autosomal reference genome by individual and variation type (B), and percent of homozygously deleted reference genome by individuals (C). The size in $B$ is calculated as the average between the haplotypes of an individual affected by the nonreference allele. Note the log-scaled y-axis in A. Per-breed bars in the histograms are stacked on each other.

We further checked for chromosome-wise differences in the number of called variants by regressing the relative number of called variants per chromosome on the relative chromosome length (Figure $S$ 3). SNPs did not show any difference to the line of identity (slope $=1.00, p=1.00$ ), while DELs (slope $=$ $1.28, \mathrm{p}=1.4 \mathrm{e}-4$ ) and INVs (slope $=1.39, \mathrm{p}=6.1 \mathrm{e}-9$ ) showed a significant bias towards larger chromosomes. DUPs (slope $=1.13, p=0.34$ ) and BNDs (slope $=1.14, p=0.17$ ) also showed a numerical bias towards larger chromosomes, which, however, was not significant. Note that the $\mathrm{R}^{2}$ value of the model was comparably small with 0.39 . 


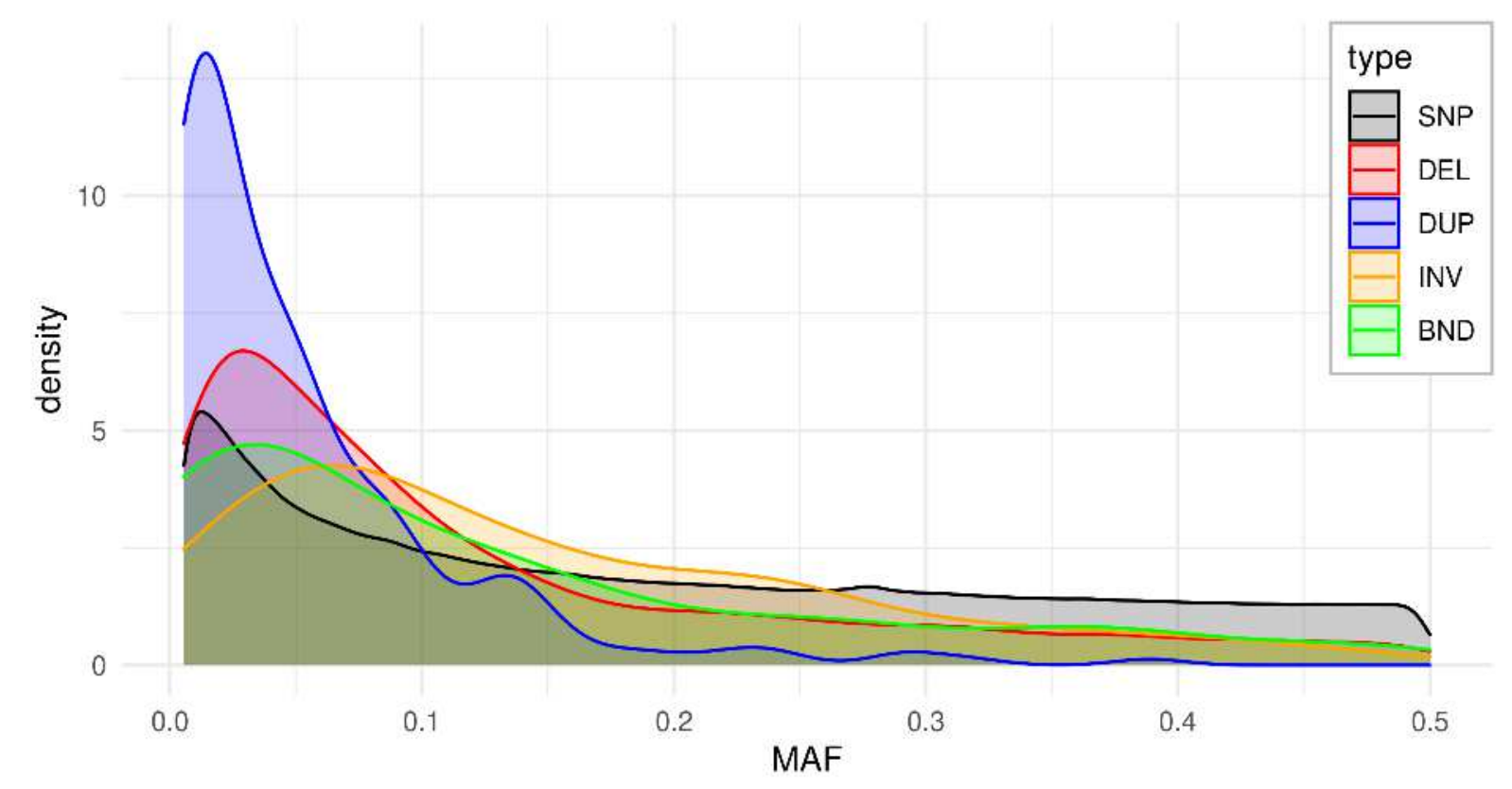

161

Distributions of minor allele frequencies (MAF; Figure 2) revealed a slight (DEL) to strong (DUP) shift towards rare variants compared with SNPs for DELs and DUPs, while INVs and BNDs showed a slight shift towards more common variants.

Figure 2: Distribution of minor allele frequency (MAF) across all samples by variant type.

Variant effect predictions of Ensembl-vep [55] classified $98.48 \%$ of the impacts of SNPs on genes as MODIFIER, $1.14 \%$ as LOW, 0.37 as MODERATE and only $0.01 \%$ as HIGH. DEL impacts were classified only in $0.41 \%$ of the cases other than MODIFIER (MODERATE $=0.01 \%$; HIGH $=0.40 \%$ ), while DUP impacts were classified as HIGH in $9.95 \%$ of the cases (MODIFIER $=90.05 \%$ ). In contrast, INV and BND impacts were completely classified as MODIFIER. Further results of VEP are summarized in Figure S 4.

\section{LD decay}

To assess the information content of SNPs on SVs, we calculated the LD between SVs and all bivariate SNPs up to $100 \mathrm{~kb}$ apart from the breakpoints as squared haplotype correlation $\left(r^{2}\right)$. Note, that SNPs 
that were located on SVs were excluded from the analysis, as their calls may be directly influenced by the SV. To get a baseline for comparisons, we also calculated the SNP - SNP LD within this distance.

Mean SNP - SNP $r^{2}$ was highest in WL (0.51 within 500 bp), followed by BL (0.41) and BR (0.26). The DEL - SNP LD decay curve follows closely the pattern of the SNP - SNP LD decay (Figure 3). Even though the level of LD was strongly reduced for the other variant types, a slight decay curve with increasing distance was still noticeable. Due to the small number of called DUPs in WL, the decay curve strongly fluctuated in this population. However, BR and BL gave some evidence that the DUP - SNP and INV SNP decay curves were comparable, while BND - SNP decay came with a slightly lower level of LD.

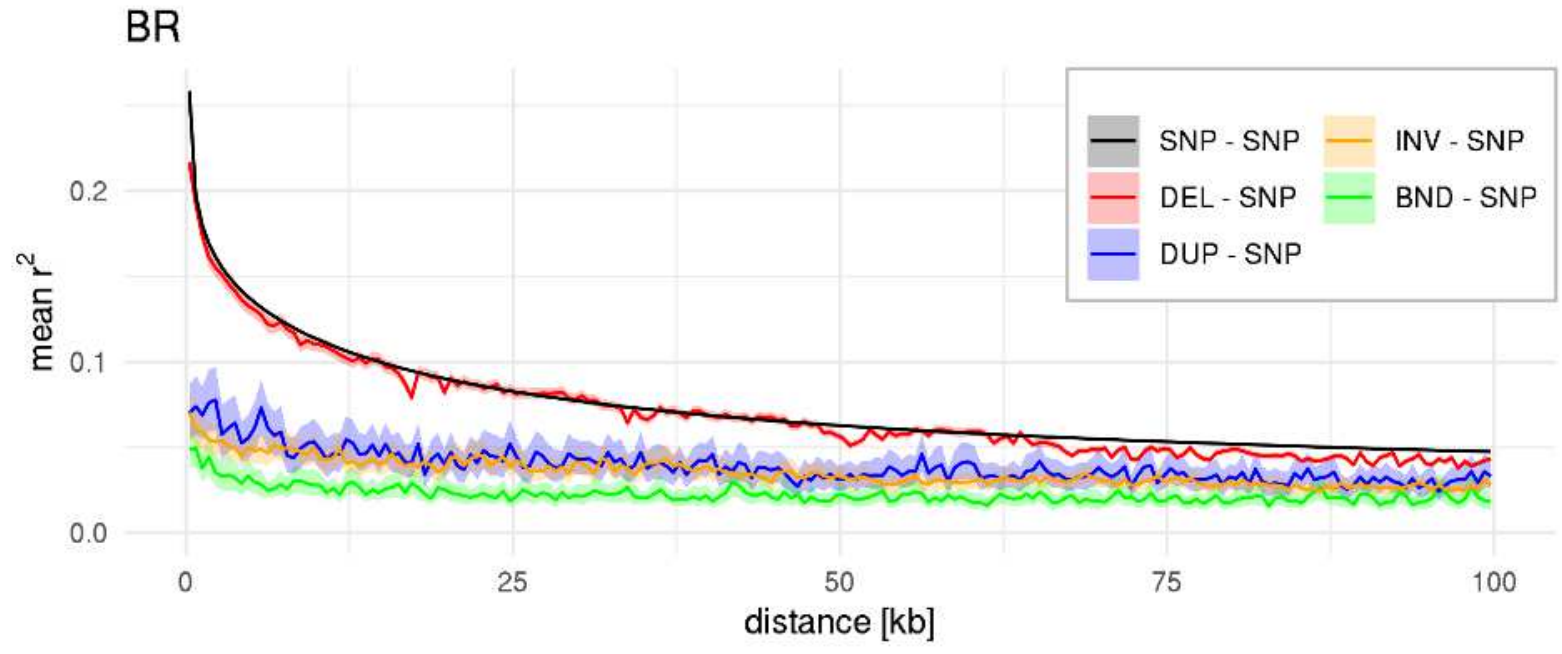

BL

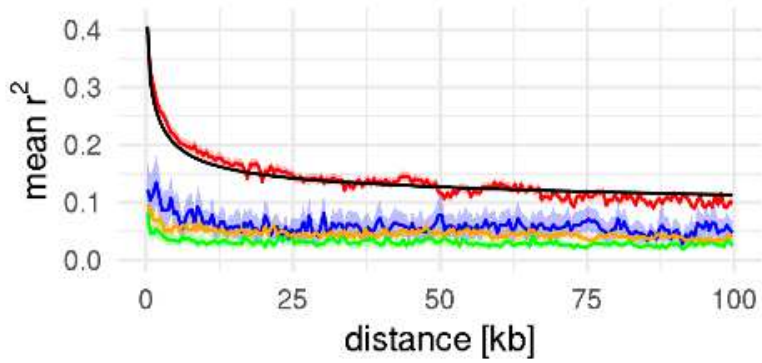

WL

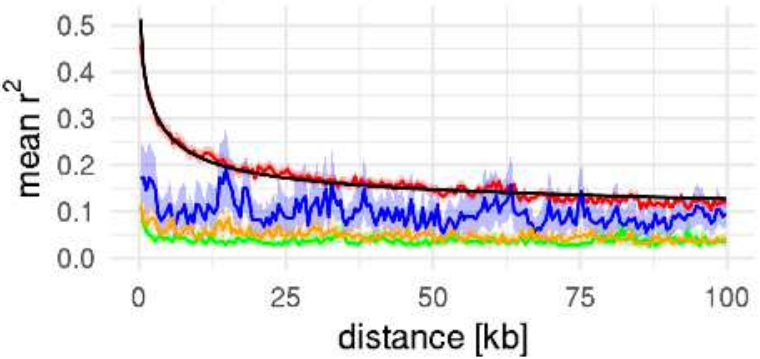

Figure 3: $L D$ decay in the broiler (BR), brown layer (BL) and white layer $(W L)$ chickens. The $L D$ is presented as mean $r^{2}$ in 500 bp distance bins and the shaded areas represent Bonferroni-corrected $95 \%$ bootstrap confidence intervals. For SNP - SNP distance bins with $>1 \mathrm{M} r^{2}$ values, no confidence intervals were estimated. 
187 To quantify the difference in LD between variants and populations and account for the population-

188 specific level of LD, we expressed the mean LD in the $500 \mathrm{bp}$ bins relative to the SNP - SNP LD and

189 further averaged those values for the first 10 bins (Table 1). This revealed comparable values within 190 variants and across populations of less than $12 \%$ difference. Across all populations, DEL - SNP LD was

191

192

193

194

195

on the same level as SNP - SNP LD, while DUP - SNP LD was $\sim 40 \%$, INV - SNP 27 \% and BND - SNP $\sim 19 \%$ of SNP - SNP LD within $5 \mathrm{~kb}$ distance. Note that the relative $r^{2}$ was not necessarily constant across the complete range of $100 \mathrm{~kb}$ (Figure S 5).

Table 1: $S V-S N P r^{2} / r_{S}^{2}$ relative to the $S N P-S N P r^{2} / r_{S}^{2}$

\begin{tabular}{|c|c|c|c|c|c|c|c|c|c|c|c|c|}
\hline \multirow{2}{*}{ Tyре } & \multicolumn{3}{|c|}{ All } & \multicolumn{3}{|c|}{ BR } & \multicolumn{3}{|c|}{$B L$} & \multicolumn{3}{|c|}{ WL } \\
\hline & $r^{2^{*}}$ & $r_{S}^{2 * *}$ & $\Delta^{* * *}$ & $r^{2^{*}}$ & $r_{S}^{2 * *}$ & $\Delta^{* * *}$ & $r^{2^{*}}$ & $r_{S}^{2 * *}$ & $\Delta^{* * *}$ & $r^{2^{*}}$ & $r_{S}^{2 *}$ & $\Delta^{* * *}$ \\
\hline DEL - SNP & $\begin{array}{c}100.1 \\
\pm 6.1\end{array}$ & $\begin{array}{c}98.8 \\
\pm 4.3\end{array}$ & -1.3 & $\begin{array}{c}95.4 \\
\pm 4.1\end{array}$ & $\begin{array}{c}94.2 \\
\pm 2.5\end{array}$ & -1.2 & $\begin{array}{c}107.0 \\
\pm 3.2\end{array}$ & $\begin{array}{c}103.2 \\
\pm 1.2\end{array}$ & -3.8 & $\begin{array}{c}98.1 \\
\pm 3.4\end{array}$ & $\begin{array}{c}98.8 \\
\pm 2.5\end{array}$ & 0.7 \\
\hline DUP - SNP & $\begin{array}{c}39.9 \\
\pm 6.8\end{array}$ & $\begin{array}{c}68.2 \\
\pm 8.9\end{array}$ & 28.3 & $\begin{array}{c}39.5 \\
\pm 5.8\end{array}$ & $\begin{array}{c}66.7 \\
\pm 4.3\end{array}$ & 27.2 & $\begin{array}{c}41.1 \\
\pm 7.0\end{array}$ & $\begin{array}{c}65.6 \\
\pm 9.9\end{array}$ & 24.5 & $\begin{array}{c}39.1 \\
\pm 8.1\end{array}$ & $\begin{array}{l}72.3 \\
\pm 10.5\end{array}$ & 33.2 \\
\hline INV - SNP & $\begin{array}{c}26.8 \\
\pm 5.2\end{array}$ & $\begin{array}{c}46.0 \\
\pm 4.3\end{array}$ & 19.2 & $\begin{array}{c}32.6 \\
\pm 2.4\end{array}$ & $\begin{array}{c}46.8 \\
\pm 1.7\end{array}$ & 14.2 & $\begin{array}{c}26.0 \\
\pm 2.4\end{array}$ & $\begin{array}{c}50.1 \\
\pm 3.4\end{array}$ & 24.1 & $\begin{array}{c}21.6 \\
\pm 3.1\end{array}$ & $\begin{array}{c}50.1 \\
\pm 6.2\end{array}$ & 28.5 \\
\hline BND - SNP & $\begin{array}{c}18.5 \\
\pm 3.6\end{array}$ & $\begin{array}{c}46.9 \\
\pm 5.4\end{array}$ & 28.4 & $\begin{array}{c}22.4 \\
\pm 2.3\end{array}$ & $\begin{array}{c}50.4 \\
\pm 3.7\end{array}$ & 28.0 & $\begin{array}{c}18.0 \\
\pm 1.9\end{array}$ & $\begin{array}{c}44.6 \\
\pm 5.1\end{array}$ & 26.6 & $\begin{array}{c}15.3 \\
\pm 2.0\end{array}$ & $\begin{array}{c}45.5 \\
\pm 5.7\end{array}$ & 30.3 \\
\hline
\end{tabular}

${ }^{*}$ Means of the first ten 500 bp bins relative to the SNP - SNP $r^{2}$ [\%] \pm standard deviations [\%]

${ }^{* *}$ Means of the first ten 500 bp bins relative to the SNP - SNP $r_{S}^{2}[\%] \pm$ standard deviations [\%]

${ }^{* * *}$ Difference between relative $r^{2}$ and relative $r_{S}^{2}$

\section{Effect of allele frequency}

Figure 2 revealed differences in the MAF spectra of the variant types. We therefore further evaluated local MAF differences ( $\triangle M A F)$ within-population by comparing $\triangle M A F$ for the SNP - SNP and SV - SNP pairs within $5 \mathrm{~kb}$ distance. This revealed elevated $\triangle M A F$ for DUP - SNP, INV - SNP, and BND - SNP pairs compared to SNP - SNP and DEL - SNP pairs in BL and WL (Figure S 7, Figure S 8), but not in BR (Figure S 6). As the upper bound of $r^{2}$ directly depends on $\triangle \mathrm{MAF}$ [20], we investigated which part of the 
observed differences in the LD decay curves is due to the observed allele frequency differences. For this, we used the standardized squared correlation coefficient $\left(r_{S}^{2}\right)$, which expresses $r^{2}$ as the proportion of the maximum possible $r^{2}$ given $\triangle M A F$ of the two variants [20] and thereby excludes effects of different allele frequencies on $r^{2}$. Mean $r_{S}^{2}$ values (Figure $\mathrm{S} 1$ ) were generally higher than mean $r^{2}$ values (Figure 3) due to the removal of the allele-frequency-dependent component. While the $r_{S}^{2}$ values of DEL - SNP relative to the SNP - SNP values (Table 1 ) were on a comparable level of $>94 \%$ as the relative $r^{2}$ values (-3.8 $\%$ to $\left.+0.7 \%\right)$, the relative $r_{S}^{2}$ values of DUPs, INVs and BNDs were between $14 \%$ and $33 \%$ higher than the according relative $r^{2}$ values. The relative $r_{S}^{2}$ values for the complete range of $100 \mathrm{~kb}$ are shown in Figure $\mathrm{S} 9$.

\section{Absence of homozygous SV genotypes}

During the investigation of the reasons for the lower level of LD between non-DEL SVs and SNPs, we realized a strong absence of homozygous calls for DUPs, INVs, and BNDs, but not for DEL (exemplarily demonstrated for BR in Figure $4 \mathrm{~A}$ ). To check whether this deviation is due to small variant allele frequencies, we calculated the deviation to Hardy-Weinberg-Equilibrium (HWE) and tested those for significance, using a Haldane Exact test under usage of the R package HardyWeinberg 1.7.2 [56] (exemplarily shown for BR in Figure 4 B). Homozygous DEL calls deviated into positive as well as into negative direction from the HWE. Homozygous calls for the other SV classes instead nearly exclusively deviated into a negative direction for all populations and only negative deviations were significant. 
A

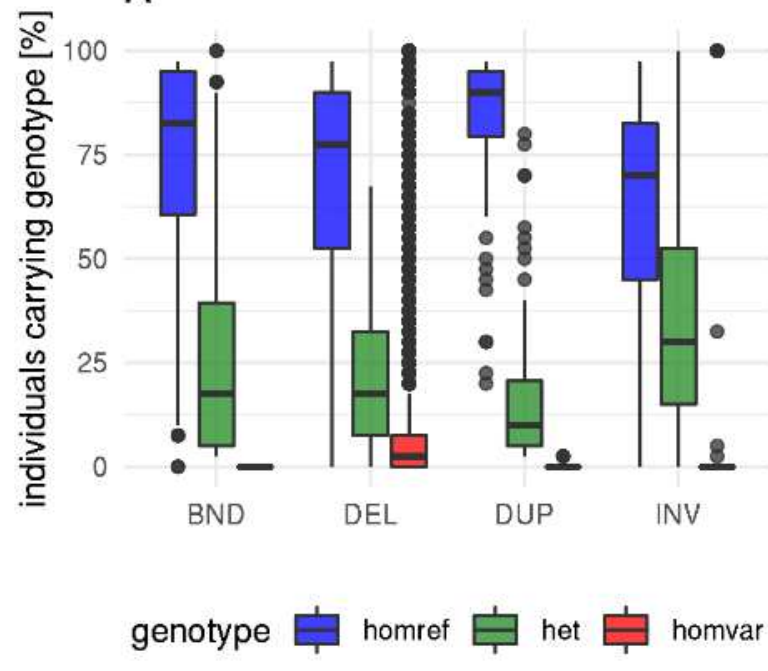

B

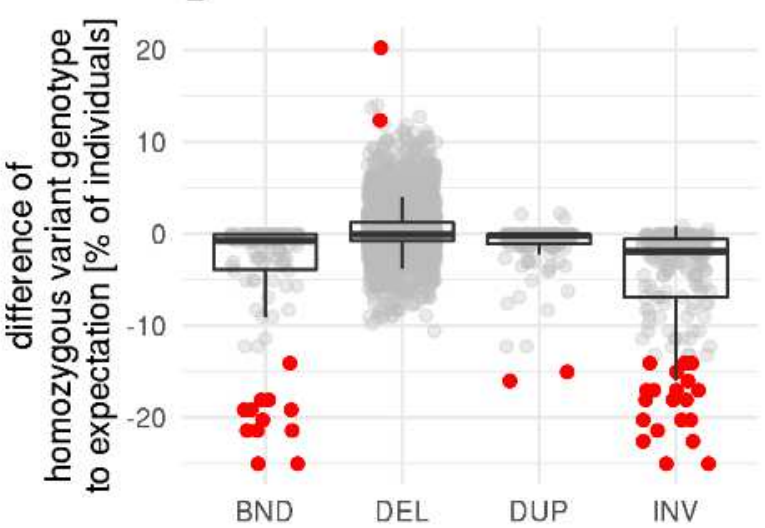

n.s. $\quad p<0.05 / \mathrm{N}$

Figure 4: Percentage of individuals carrying SV genotype (A) and deviations of homozygous variant genotypes from the Hardy-Weinberg-Expectation (B) in the broiler population for each called SV. Deviations from HWE were tested by a Haldane Exact test under usage of the R package HardyWeinberg 1.7.2 [56]. Bonferroni correction of the $p$ values was applied within SV class. Homref-homozygous for the reference allele; het - heterozygous; homvar - homozygous for the variant allele; n.s. - not significant. Comparable figures for WL and BL can be found in Figure S 11 and Figure S 12.

We tried to tackle the effect of this problem by correlating the 0/1/2 coded SNP genotypes with a coverage-dependent measure of copy number for DELs and DUPs, the Duphold Flanking Fold Change (DHFFC) [57]. However, as the DHFFC was also used for filtering, the results of this are potentially confounded and are only part of the supplementary material (Supplementary File 1).

\section{Taggability}

Theoretically, one SNP in strong LD to the variant of interest would be enough to serve as a marker that (partly) captures the effect of the variant for, e.g., GWAS or genomic selection as tag SNP. We, therefore, investigated the presence of potential tag SNPs close to the variants of interest. The used measure is the maximum observed $r^{2}$ between a variant of interest and a pool of potential tag SNPs within a certain distance $\left(r_{t a g}^{2}\right)$. Nearly all variants in all variant classes came with at least one variable SNP within proximity of $10 \mathrm{~kb}$ (Figure S 14). Mean $r_{\text {tag }}^{2}$ for all variants and populations showed an 
asymptotic trend with identifying the best tag SNP within $10 \mathrm{~kb}$ for most of the variants in all three populations (Figure 5). Only mean $r_{\text {tag }}^{2}$ of DUPs in BR was continuously growing until $100 \mathrm{~kb}$ distance (Figure 5). Mean $r_{\text {tag }}^{2}$ for SNPs only reached $\sim 0.9$ within $100 \mathrm{~kb}$ in all three populations, meaning that some SNPs were not in full phase to any other SNP. Mean $r_{\text {tag }}^{2}$ was slightly reduced for DELs and strongly for DUPs, INVs and BNDs compared to SNPs (Figure 5).
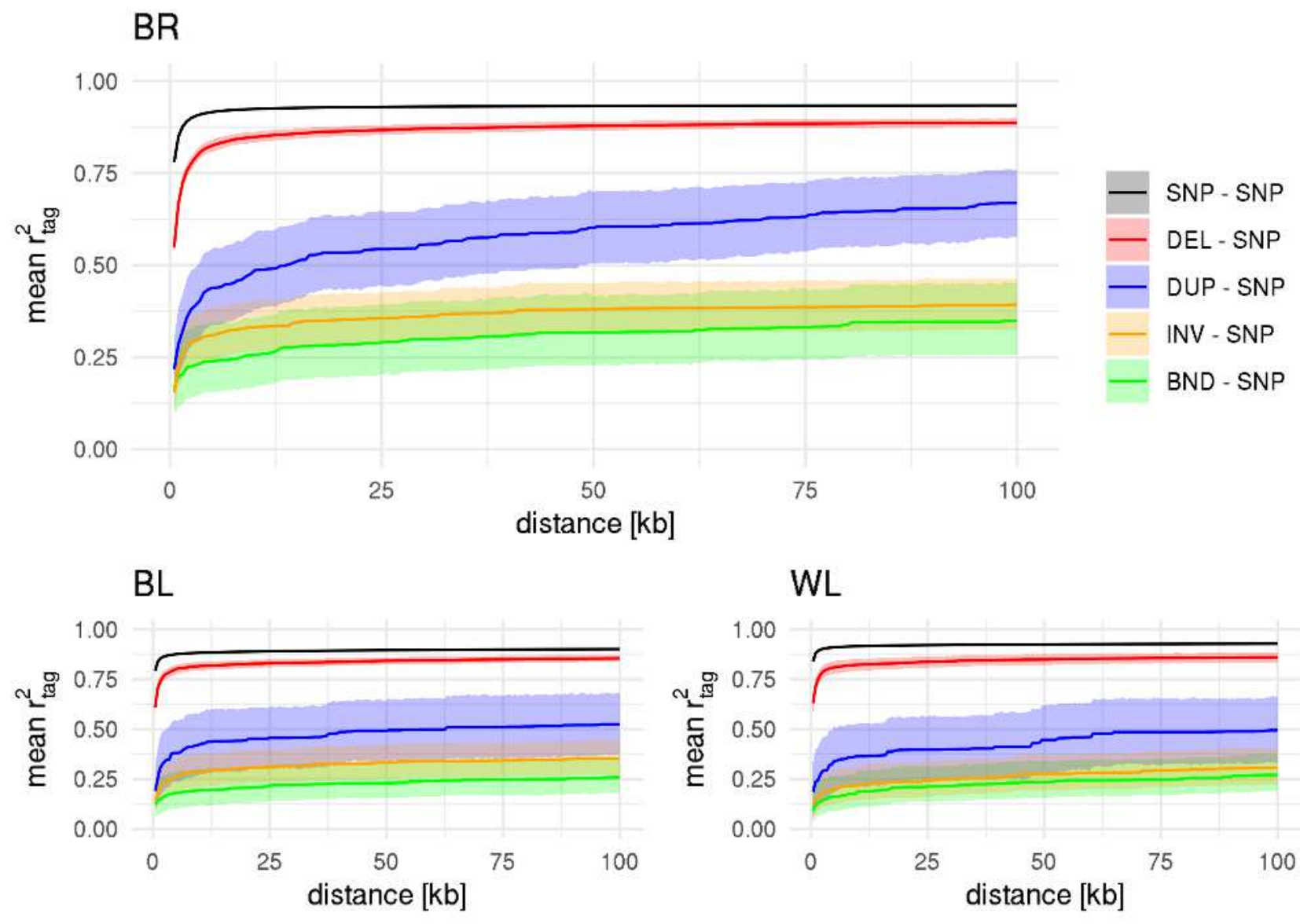

Figure 5: Mean taggability for broiler (BR), brown layer (BL), and white layer (WL) chickens. Taggability $\left(r_{\text {tag }}^{2}\right)$ was calculated as the maximum $r^{2}$ value up to a certain distance from the variant of interest. Means across variants are presented as lines while the shaded area represents the Bonferroni-corrected $95 \%$ bootstrap confidence intervals. variants. While more than $85 \%$ of the SNPs were tagged in BR within $10 \mathrm{~kb}$, this number was slightly 
smaller for DELs (>75\%). More than $25 \%$ of the DUPs were tagged within $10 \mathrm{~kb}$ distance and $50 \%$ within $100 \mathrm{~kb}$, while less than $15 \%$ of INVs and BNDs were tagged. The tendency is the same in the two layer populations, but the absolute numbers slightly deviate. As a maximum value of a sample is not independent of the number of sampled values, we also checked the number of present potential tag SNPs within $5 \mathrm{~kb}$ distance to the variant of interest. Interestingly, SNPs were surrounded by significantly more close variable SNPs on average than SVs in all three populations (Table 2). This difference was still present when regarding only tag SNPs $\left(r^{2}>0.75\right)$.

Table 2: Median number of variable SNPs within $5 \mathrm{~kb}$ distance to variants of interest

\begin{tabular}{lcccccccc}
\hline \multirow{2}{*}{ Variant } & \multicolumn{2}{c}{ BR } & & \multicolumn{2}{c}{ BL } & & \multicolumn{2}{c}{ WL } \\
\cline { 2 - 5 } \cline { 7 - 8 } & All & $r^{2} \geq 0.75$ & & all & $r^{2} \geq 0.75$ & & all & $r^{2} \geq 0.75$ \\
\hline SNP & $140^{\mathrm{a}}$ & $7^{\mathrm{a}}$ & & $85^{\mathrm{a}}$ & $5^{\mathrm{a}}$ & & $73^{\mathrm{a}}$ & $9^{\mathrm{a}}$ \\
DEL & $70^{\mathrm{d}}$ & $5^{\mathrm{b}}$ & & $41^{\mathrm{c}}$ & $4^{\mathrm{b}}$ & & $38^{\mathrm{c}}$ & $6^{\mathrm{b}}$ \\
DUP & $78^{\mathrm{cd}}$ & $4^{\mathrm{b}}$ & & $48^{\mathrm{bc}}$ & $3^{\mathrm{ab}}$ & & $31^{\mathrm{c}}$ & $4^{\mathrm{ab}}$ \\
INV & $90^{\mathrm{c}}$ & $3^{\mathrm{b}}$ & & $49^{\mathrm{bc}}$ & $5^{\mathrm{ab}}$ & & $42^{\mathrm{bc}}$ & $11^{\mathrm{ab}}$ \\
BND & $119^{\mathrm{b}}$ & $6^{\mathrm{ab}}$ & & $59^{\mathrm{b}}$ & $1^{\mathrm{ab}}$ & & $61^{\mathrm{b}}$ & $1^{\mathrm{ab}}$ \\
\hline
\end{tabular}

Different lowercase letters within columns account for significantly different medians at the significance level of 0.05 (Bonferroni-corrected pairwise Wilcoxon rank-sum test)

In practice, the interest of researchers and breeding companies may not be the taggability of SVs by WGS SNPs, but by array SNPs. Those come with a different allele frequency spectrum and lower resolution than WGS SNPs, which influences the LD patterns [19]. However, they are often available for a huge number of phenotyped individuals due to their use in routine breeding programs. We, therefore, evaluated the potential performance of four publically available chicken genotyping arrays with resolutions of $600 \mathrm{k}$ [58], $60 \mathrm{k}$ [59], $55 \mathrm{k}$ [60], and $10 \mathrm{k}$ [61].

The availability of variable SNPs close to the variants of interest was strongly dependent on the resolution of the arrays. While the $600 \mathrm{k}$ array had a variable array SNP within $15 \mathrm{~kb}$ for more than $90 \%$ of 
the variants in all three populations, the $60 \mathrm{k}$ and the $55 \mathrm{k}$ array came with a slight shift of this dependency of having a variable array SNP for $>80 \%$ of the variants at $50 \mathrm{~kb}$ and $>90 \%$ at $100 \mathrm{~kb}$ (Figure S 16). The $10 \mathrm{k}$ array, however, contained no variable array SNP for $50 \%$ of the variants within $100 \mathrm{~kb}$. A non-random difference in SNP density by variant type is not present for any array. The reduced density compared to WGS also reduced the taggability. Mean $r_{\text {tag }}^{2}$ values for SNPs and DELs reached between 0.06 for BR and the $10 \mathrm{k}$ array and 0.65 for WL and the $600 \mathrm{k}$ array within $100 \mathrm{~kb}$ distance (Figure S 15). Interestingly, DELs seem to be slightly stronger tagged than SNPs in BL and WL (Figure S 15), while the other variant types were tagged by maximally $50 \%$ of the level which was reached in SNPS and DELs. The results are comparable when checking the proportion of variants with $r_{\text {tag }}^{2}>0.75$ (Figure S 17). $40 \%$ of the WGS SNPs and even $45 \%$ of DELs were tagged with more than $r_{\text {tag }}^{2}>0.75$ by a SNP of the $600 \mathrm{k}$ array in WL. In contrast, less than $1 \%$ of SNPs and DELs were tagged by a SNP of the 10k array in $\mathrm{BR}$.

\section{Discussion}

\section{Implications from the SV calling pipeline}

The SV calling approach was intended to return highly accurate variant calls, therefore prioritizing precision over sensitivity. This especially required the exclusion of regions with unusually high coverage, as they may be artefacts of inaccurate read mapping in regions of low sequence complexity [62]. As those regions are known to be hot spots for SV formation by non-allelic homologous recombination (NAHR) [28-30,63], we expect to have missed a significant proportion of SVs, especially multi-copy DUP. Further, there was a missing overlap between DELLY and MANTA at INS calling, resulting in no INS calls. A generally weak power in INS calling from short reads is expected, though [14]. Those two problems highlight the need for long-read sequencing data for future studies, which should allow for improved resolution of complex regions and comes with improved abilities for INS calling [1, 15]. The limitations of the calling approach and the resulting characteristics of the callset need to be considered 
when comparing our results to SV callsets that were derived by different approaches and therefore probably capturing SVs with different properties.

We further identified a lack of homozygous calls of DUPs, INVs, and BNDs with regard to HWE (Figure 4, Figure S 11, Figure $S$ 12). One possible reason may be a deleterious load and therefore purifying selection on those variants. While literature highlights the deleterious potential of DELs, INVs, and BNDs $[2,64]$, DUPs are rather considered positive by increasing gene expression $[2,3]$. In our case, DELs rather show a slight excess of homozygotes than an expected lack under purifying selection (Figure 4, Figure S 11, Figure S 12). The lack of homozygous calls was instead present for DUPs, INVs, and BNDs. Additionally, VEP impact predictions classified $99.6 \%$ of the DEL impacts as MODIFIER and only $0.4 \%$ as HIGH, while DUP impacts were classified as HIGH in $10 \%$ of the cases. The discrepancy with literature for DELs may partly be due to past inbreeding in the populations $[52,65]$, which resulted in small effective population sizes [66] and therefore may have purged strongly deleterious DELs [67, 68]. Purging of deleterious DELs may, together with limitations of the used SV callers, also be a reason for the relatively short sizes of the called DELs. Nevertheless, as none of the INVs and BNDs had predicted impacts besides MODIFIER, a second reason seems to be more likely: There may be deficits of the genotypers in accurately distinguishing between heterozygous and homozygous calls of DUPs, INVs, and BNDs.

\section{LD decay results}

The overall levels of SNP - SNP LD within the populations reflect the knowledge from the literature $[19,66]$ and the different levels of variability $(B R>B L>W L)[52,69]$. This resulted in $W L$ having the strongest overall level of LD and BR the weakest. Besides that and if not especially indicated differently, results were the same for all three populations throughout the following sections.

The DEL - SNP LD, all in all, was on the same level as SNP - SNP LD. This implies good predictability of DEL effects by SNP call sets and is in accordance with the majority of the existing studies [6, 21-24]. Studies that found DEL - SNP LD to be on a reduced level compared to SNP - SNP LD mostly performed 
the DEL calling from SNP arrays, which implies low breakpoint resolution [32]. It is also common to merge CNV to copy number variable regions (CNVR) in SNP array or read-depth-based studies [32]. Therefore, a CNVR can reflect multiple mutation events and not only a single variant, resulting in reduced LD to bivariate SNPs, an effect we do not expect to be present in our data due to the more precise variant definition.

The level of DUP - SNP LD was strongly reduced compared to SNP - SNP LD and DEL - SNP LD, which is in accordance with the existing studies $[22,26,28,32]$. However, levels of $\sim 40 \%$ of the SNP - SNP LD (Table 1) were higher than what was found e.g. by Lee et al. [32], who found DUP - SNP LD to be $\sim 20 \%$ of SNP - SNP LD in two dairy cattle populations. A main factor of DUP - SNP LD being reduced compared to SNP - SNP LD may be due to the lower allele frequencies of DUP in our callset (Figure 2) and therefore increased local $\triangle M A F$ (Figure $S 7$, Figure $S 8$ ) in BL and WL. Removing the $\triangle M A F$ dependent part of LD by expressing LD as $r_{S}^{2}$ increased the relative $r^{2}$ of $30 \%$ to a relative $r_{S}^{2}$ of $68 \%$ of the SNP $-\mathrm{SNP} r_{S}^{2}(+28 \%$,Table 1$)$. This means that local differences in the allele frequency spectra between SNPs and DUP account for $\sim 50 \%$ of the difference between SNP - SNP LD and DUP - SNP LD

A second cause for reduced DUP - SNP LD could be a higher rate of genotyping errors in DUP. In fact, we identified a significant reduction of homozygous DUP calls compared to HWE (Figure 4, Figure S 11, Figure S 12) as already discussed above. The potential genotyping inaccuracy may additionally be supported by the, admittedly subjective, observation of the two assessors during the visual filtering step that DUP came with less clear support than DEL. This, however, resulted only in a moderately reduced inter-observer reliability of $94 \%$ in DUP compared to $97 \%$ in DEL (Supplementary file 3).

A further possibility of reduced DUP - SNP LD may be the occurrence of multi-copy CNVs (mCNVs) [28, 30] in our callset. DUP in the callset may partly represent CNVs that occur with different copy numbers and are therefore multi- instead of bivariate variants. This reduces the linkage to bivariate SNPs. We saw slight support for the occurrence of some mCNV in the callset e.g. by some high DHFFC values. 
However, mCNVs are known to cluster in special regions of the genome [28] due to non-allelic homologous recombination (NAHR) as a formation mechanism $[29,70]$. Note that NAHR can also occur recurrently [29], resulting in variants that are called bivariate but stem from multiple mutation events. As those clusters should result in high-coverage regions, which we removed in the filtering step, we do not expect a higher number of mCNV and recurrent mutations in our callset.

We also evaluated the linkage between SNPs and INV/ BND and found low levels of LD $(26.8 \%$ and $18.5 \%$ of SNP - SNP LD). The reduced LD in our study is again partly due to local allele frequency differences (Figure S 6 - Figure S 8) as for DUP. Relative $r_{S}^{2}$ values were therefore $14 \%$ to $30 \%$ higher than relative $r^{2}$ values (Table 1 ). However, $r_{S}^{2}$ values for INV - SNP and BND - SNP were still only $50 \%$ of SNP - SNP $r_{S}^{2}$. The remaining gap may partly be due to genotyping problems. We identified the lack of homozygous calls for INVs and BNDs (Figure 4, Figure S 11, Figure S 12) as for DUPs. In combination with the missing ability to use coverage information for filtering, we would trust the INV and BND genotypes least in our callset. In contrast to our results, Sudmant et al. [28] found INV to be in good LD to SNPs in a very accurate callset from 2,504 human genomes, which further supports that the accuracy of INV calls was low in our study.

\section{Taggability}

The analysis of taggability revealed comparable patterns as the LD decay. A high fraction of SNPs and DEL was tagged by close-by WGS SNPs in all three populations (Figure 5; Figure S 14), while only a small fraction of DUPs, INVs, and BNDs was tagged. However, in contrast to the decay patterns, SNPs on average were tagged slightly stronger than DEL, and between $5 \%$ and $10 \%$ more SNPs were tagged with $r_{\text {tag }}^{2}>0.75$ than DEL. A reason for the higher taggability of SNPs compared to DEL, while the LD decay does not differ, may be the reduced SNP density around DELs (Table 2), as the chance for higher maximum values increases with the number of SNPs in the region of interest. In contrast, DELs were tagged slightly better by array SNPs than WGS SNPs by array SNPs. In the case of array SNPs, no locally increased density was present, as array design aims at an equidistant spacing of markers across the genome [58]. This resulted in no difference between the taggability of SNPs and DELs by array SNPS. 
Potential issues of excluding SNPs in complex regions during array design as suggested by Lee et al.

[32] as a reason for reduced CNV - SNP LD, were not observed in this study, as we excluded SVs in

380 those regions due to a minor calling accuracy. Using array SNPs to tag the WGS variants further revealed a strong need for dense marker maps to provide good tag SNPs, as only the $600 \mathrm{k}$ array could provide tag SNPs with $r_{\text {tag }}^{2}>0.75$ for more than $25 \%$ of SNPs and DEL. This may largely explain why cattle to be not tagged by SNPs of a $50 \mathrm{k}$ array. It suggests that this is not solely due to the nature of CNV but that they also missed a comparable fraction of effects, which are caused by SNPs.

The concept of taggability is especially relevant for GWAS, where phenotype-marker associations are tested for each marker separately. The strength of the LD between marker and causal variant then directly influences the power of the GWAS. However, the absence of single tag SNPs does not imply that the effect of an SV cannot be captured by a longer haplotype. Methods that utilize effects of multiple SNP at once (e.g. ridge regression best linear unbiased prediction [71]), of which each can explain a slightly different fraction of the variance of the causal variant, may be more robust in this sense. Additionally, imputation of known SVs would probably be a way to overcome the issue of low taggability and needs further investigation.

\section{Conclusions}

We evaluated LD patterns between a comprehensive SV callset and surrounding SNPs in three commercial chicken populations. We found DEL - SNP LD to be on the same level as SNP - SNP LD, while DUP - SNP, INV - SNP, and BND - SNP LD were strongly reduced. This was in accordance with the availability of tag SNPs for a high share of SNPs and DELs, while tag SNPs for DUPs were rare and mostly missing for INVs and BNDs. Different arrays came with a density-dependent ability to tag WGS SNPS and SVs but did not show strong systematic differences compared with taggability by WGS SNPs. The main reason for existing differences in SNP - SNP and DUP/INV/BND - SNP LD in our study was due to local MAF differences. Those accounted for $\sim 50 \%$ of this difference in the strength of LD. This implies 
that genomic variance due to DELs in the chicken populations studied can be captured by different SNP marker sets as good as variance from WGS SNPs, whereas separate SV calling might be advisable for DUP, INV, and BND effects.

\section{Material and Methods}

\section{Data}

The study used WGS data of 25 white layers, 25 brown layers, and 40 broiler chickens. The raw data was first published by Qanbari et al. [52], which contains more information about the samples. Chickens were paired-end sequenced with a median coverage between $5 \mathrm{X}$ and $17 \mathrm{X}$, read length of $100 \mathrm{bp}$ $(\mathrm{WL}+\mathrm{BL})$ or $126 \mathrm{bp}(\mathrm{BR})$, and insert sizes of $\sim 400 \mathrm{bp}$. Basic quality statistics can be found in Supplementary file 2 as MultiQC report [72].

Population integrity was controlled using principal component analysis in plink 1.9 [73]. The SNPs were first LD pruned by setting the --indep-pairwise flag to sliding windows of $50 \mathrm{~kb}$, a stepsize of five SNPs and an $r^{2}$ of 0.5 . Based on the pruned SNPs, plink extracted then 90 prime components. Results for the first four prime components and the variance explained can be found in Figure $S$ 18. The first two prime components, which in total accounted for $33.2 \%$ of the total variance, clearly separated broilers, white- and brown layers. The two broiler subpopulations were only slightly separated by the second prime component and clearly by the third, which accounted for $4.5 \%$ of the total variance. The fourth component started splitting one of the broiler populations. We assumed this to be sufficiently closely related to consider the two broiler subpopulations as a combined population for further analyses.

\section{Variant Calling Pipeline}

Alignment on the reference genome galGal6/ GRGC6a and SNP calling were conducted in a previous study [53] following GATK best practices pipeline [74]. The SNPs needed for this study were then extracted from the old callset using bcftools [75] and the duplicate-marked and base quality score recalibrated BAM files were used as starting point for the SV calling process. 
SV calling was conducted following a consensus calling approach. SVs were first separately called per

428 individual and then genotyped on population-level by running Delly 0.8.5 [10], Manta 1.6.0 [12], and a

429 combination of Lumpy 0.2.13 [11] and Svtyper 0.7.0 [76] in parallel on the complete set. The genotyp-

430 ing results of the three calling pipelines were then merged using SURVIVOR 1.0.7 [77] and allowing for

431 breakpoint differences of $1000 \mathrm{bp}$. This resulted in 95,478 raw SV calls.

Additionally, read depth profiles for all samples in 100 bp windows were generated using Mosdepth 0.2.9 [78] and SVs were annotated with Dupholds (version 0.2.1) [57] flanking fold change (DHFFC) and the SNP genotype calls located on the SV.

The merged callset was then filtered based on the following parameters:

1) Caller overlap: At least two of the three callers needed to support the variant.

2) Genotype concordance: The genotype that was supported by two out of the three callers was considered as the consensus genotype. Genotypes without the necessary support were set to missing for later re-imputation. If more than two samples did not have the necessary genotype concordance support for an SV, the complete SV was removed from the data set.

3) Removal of high coverage regions: Local coverage was extracted by Mosdepth 0.2 .9 [78] in 100 bp windows. If windows exceeded a threshold of twice the average coverage across all samples (expected value for a fixed DUP) plus two standard deviations, they were classified as unusually highly covered. Unusually highly covered regions were further merged if they were less than $1000 \mathrm{bp}$ apart from each other. SVs with breakpoint confidence intervals falling in such a region were removed from the data set.

4) Difference to flanking coverage: DELs and DUPs calls were checked for non-consistent coverage changes relative to the flanking coverage by evaluating the Duphold Flanking Fold Change (DHFFC) [57]. DELs were considered as wrong genotypes when heterozygotes were not between 0.1 and 0.9 and homozygous DEL genotypes not smaller than 0.25 . Heterozygous DUPs had to be $>1.1$ and homozygous DUPs $>1.5$. DELs/DUPs with more than one error or more than 

$10 \%$ wrong genotypes were filtered. Otherwise, the putatively wrong DEL/DUP genotypes were set to missing for later re-imputation.

5) Support by SNP calls on DELs: SNP calls need to be homozygous on heterozygous DELs and missing on homozygous DELs. We, therefore, calculated for each DEL genotype the relative number of wrong SNP genotypes (e.g. one error by five total SNPs on the DEL $=0.1$ ). If the sum of those error rates across samples exceeded two or $50 \%$ of the number of samples that were at least heterozygous for the DEL, the DEL was filtered. Otherwise, the putatively wrong DEL genotypes were set to missing for later re-imputation.

This resulted in 5,600 SVs (4,831 DELs; 253 DUPs; 346 INVs; 170 BNDs; $94.1 \%$ filtered). No INS remained, as Lumpy does not call INS and there was no overlap between Delly and Manta. Samplot 1.0.19 [54] was then used to generate quality control plots for each SV that passed the previous filtering step. The quality plots were visually screened by two separate observers comparable to the workflow implemented in SV-plaudit [79], but implemented locally by using image-sorter2 (https://github.com/Nestak2/image-sorter2). The SVs needed to be scored as 'pass' by each of the two observers to be further used (Supplementary file 3). By this, a further $6.9 \%$ of the SVs (3.5 \% of DEL, $11.1 \%$ of DUP, $36.0 \%$ of INV, and $30.8 \%$ of BND) were removed. The removed SVs were mainly in regions with complex mapping patterns.

The final SV callset (4,301 DEL, 224 DUP, 218 INV, 117 BND) was then merged with the SNP callset $(12,294,329$ bivariate autosomal SNPs). The samples were phased and missing genotypes were imputed by beagle 5.0 [80] with default settings besides reducing 'ne' to 10,000 [81]. Functional consequences were annotated by ensembl-vep [55] using the release 100 GRGC6a annotation files.

\section{Estimation of LD}

LD between two loci with a maximum distance of $100 \mathrm{~kb}$ was initially estimated from phased haplotypes as follows: 


$$
r_{A B}^{2}=\frac{\left(p_{A B}-p_{A} p_{B}\right)^{2}}{p_{A} p_{B}\left(1-p_{A}\right)\left(1-p_{B}\right)}
$$

477 Where $p_{A}$ and $p_{B}$ account for the alternative allele frequencies at the two loci and $p_{A B}$ for the accord478 ing haplotype frequency. To control for allele frequency deviations that influence the maximum possi479 ble $r^{2}$, we further scaled $r^{2}$ by the maximum possible $r^{2}$ given $\triangle M A F\left(r_{S}^{2}=r^{2} / r_{\max \mid \Delta M A F}^{2}\right)$ where $480 \quad r_{\max \mid \triangle M A F}^{2}$ was derived as described by VanLiere and Rosenberg [20]. As we realized a problem with 481 calling of homozygous DUP, we additionally estimated LD as squared Pearson Correlation between 0/1/2 coded SNP genotypes and the Duphold Flanking Fold Change (DHFFC) [57] as a measure for the relative reference genome coverage at DEL and DUP (due to possible confounding only part of Supplementary file 1). LD decay was then summarized in means of $500 \mathrm{bp}$ bins between the variants.

Bonferroni corrected bootstrap confidence intervals for the LD decay were estimated by resampling the $r^{2}$ values within each bin 100,000 times with replacement. As tests showed confidence intervals for SNP - SNP LD being $<0.001$ due to the huge number of underlying values, we decided to skip estimation of confidence intervals for bins with $>1 \mathrm{M} r^{2}$ values.

A tag SNP was defined as the SNP with the highest $r^{2}$ to the variant of interest within a certain distance $\left(r_{t a g}^{2}\right)$. The taggability of variant classes was then investigated by comparing means of $r_{\text {tag }}^{2}$ and shares of variants with $r_{\text {tag }}^{2}>0.75$. Additionally to the taggability by WGS SNPs, we compared the taggability by SNPs of four commercially available SNP arrays. The $600 \mathrm{k}$ Affymetrix Axiom chicken genotyping array [58], a 60 k Illumina Bead Chip [59], a 55 k Affymetrix genotyping array [60], and the IMAGE_001 multispecies array, which contains $10 \mathrm{k}$ chicken-specific SNPs on an Affymetrix genotyping array [61]. The annotation files were lifted over to the reference genome galGal6/GRGC6a by the UCSC [82] liftdefined as pools of potential Array tag SNPs. 


\section{Workflow}

The complete pipeline was set up in snakemake 5.3.0 [83] and the according scripts including the snakefile with all used parameters as well as the dependency analytics graph (DAG) and the rulegraph of the pipeline can be found in the supplementary material (Supplementary file 4).

\section{Declarations}

\section{Ethics approval and consent to participate}

The study did not involve new treatment of animals as only published data was used. DNA samples for all already published raw data were taken from a database established during the project AVIANDIV (EC Contract No. BIO4-CT98_0342; 1998 - 2000; https://aviandiv.fli.de/) and later extended by samples of the project SYNBREED (FKZ 0315528E; 2009 - 2014; www.synbreed.tum.de). Blood sampling was done in strict accordance to the German animal welfare regulations, with written consent of the animal owners and was approved by the at the according times ethics responsible persons of the Friedrich-Loeffler-Institut. According to German animal welfare regulations, notice was given to the responsible institution, the Lower Saxony State Office for Consumer Protection and Food Safety (33.942502-05-10A064).

\section{Consent for publication}

Not applicable

\section{Availability of data and materials}

The raw fastq files were already published by Qanbari et al. [52] and can be accessed via the ENA project PRJEB30270 (https://www.ebi.ac.uk/ena/browser/view/PRJEB30270). The snakemake workflow together with all scripts and supplementary files can be found in Supplementary file 4. Intermediate results are available from the corresponding author on reasonable request.

\section{Competing interests}

The authors declare that they have no competing interests. 
523 We acknowledge support by the Open Access Publication Funds of the University of Goettingen.

524

525

526

527

528

529

530

531

532

533

534

535

536

537

538

539

540

541

542

\section{Authors' contributions}

JG designed the study, analyzed the data, interpreted the results, and wrote the initial manuscript. NPP substantially contributed to the data analysis. SW contributed to the acquisition of the data and revision of the manuscript. HS and CR contributed to design of the study, interpretation of the results, and substantially revised the manuscript. All authors have read and approved the submitted manuscript.

\section{Acknowledgments}

Not applicable

\section{Supplementary}

Supplementary file 1: Supplementary results, tables and figures.

Supplementary file 2: MultiQC report.

Supplementary file 3: Observer concordance of the visual filtering step.

Supplementary file 4: Snakemake workflow and scripts.

\section{References}

1. Ho SS, Urban AE, Mills RE. Structural variation in the sequencing era. Nature Reviews Genetics 2019. doi:10.1038/s41576-019-0180-9.

2. Feuk L, Carson AR, Scherer SW. Structural variation in the human genome. Nat Rev Genet. 2006;7:85-97. doi:10.1038/nrg1767.

3. Lee Y-L, Takeda H, Moreira GCM, Karim L, Mullaart E, Coppieters W, et al. A 12 kb multi-allelic copy number variation encompassing a GC gene enhancer is associated with mastitis resistance in dairy cattle; 2021. 
4. Imsland F, Feng C, Boije H, Bed'hom B, Fillon V, Dorshorst B, et al. The Rose-comb mutation in chickens constitutes a structural rearrangement causing both altered comb morphology and defective sperm motility. PLoS Genet. 2012;8:e1002775. doi:10.1371/journal.pgen.1002775.

5. Rubin C-J, Megens H-J, Martinez Barrio A, Maqbool K, Sayyab S, Schwochow D, et al. Strong signatures of selection in the domestic pig genome. Proc Natl Acad Sci U S A. 2012;109:19529-36. doi:10.1073/pnas.1217149109.

6. McCarroll SA, Hadnott TN, Perry GH, Sabeti PC, Zody MC, Barrett JC, et al. Common deletion polymorphisms in the human genome. Nature Genetics. 2006;38:86-92. doi:10.1038/ng1696.

7. Conrad DF, Andrews TD, Carter NP, Hurles ME, Pritchard JK. A high-resolution survey of deletion polymorphism in the human genome. Nature Genetics. 2006;38:75-81. doi:10.1038/ng1697.

8. Wang K, Li M, Hadley D, Liu R, Glessner J, Grant SFA, et al. PennCNV: an integrated hidden Markov model designed for high-resolution copy number variation detection in whole-genome SNP genotyping data. Genome Res. 2007;17:1665-74. doi:10.1101/gr.6861907.

9. Abyzov A, Urban AE, Snyder M, Gerstein M. CNVnator: an approach to discover, genotype, and characterize typical and atypical CNVs from family and population genome sequencing. Genome Res. 2011;21:974-84. doi:10.1101/gr.114876.110.

10. Rausch T, Zichner T, Schlattl A, Stütz AM, Benes V, Korbel JO. DELLY: structural variant discovery by integrated paired-end and split-read analysis. Bioinformatics. 2012;28:i333-i339. doi:10.1093/bioinformatics/bts378.

11. Layer RM, Chiang C, Quinlan AR, Hall IM. LUMPY: a probabilistic framework for structural variant discovery. Genome Biology. 2014;15:R84. doi:10.1186/gb-2014-15-6-r84.

12. Chen X, Schulz-Trieglaff O, Shaw R, Barnes B, Schlesinger F, Källberg M, et al. Manta: rapid detection of structural variants and indels for germline and cancer sequencing applications. Bioinformatics. 2016;32:1220-2. doi:10.1093/bioinformatics/btv710. 
13. Escaramís G, Docampo E, Rabionet R. A decade of structural variants: description, history and methods to detect structural variation. Brief Funct Genomics. 2015;14:305-14. doi:10.1093/bfgp/elv014.

14. Delage WJ, Thevenon J, Lemaitre C. Towards a better understanding of the low recall of insertion variants with short-read based variant callers. BMC genomics. 2020;21:762. doi:10.1186/s12864020-07125-5.

15. Sedlazeck FJ, Lee H, Darby CA, Schatz MC. Piercing the dark matter: Bioinformatics of long-range sequencing and mapping. Nature Reviews Genetics. 2018;19:329-46. doi:10.1038/s41576-0180003-4.

16. Auton A, Abecasis GR, Altshuler DM, Durbin RM, Bentley DR, Chakravarti A, et al. A global reference for human genetic variation. Nature. 2015;526:68-74. doi:10.1038/nature15393.

17. Hayes BJ, Daetwyler HD. 1000 Bull Genomes Project to Map Simple and Complex Genetic Traits in Cattle: Applications and Outcomes. Annual Review of Animal Biosciences. 2019;7:89-102. doi:10.1146/annurev-animal-020518-115024.

18. Malomane DK, Simianer H, Weigend A, Reimer C, Schmitt AO, Weigend S. The SYNBREED chicken diversity panel: A global resource to assess chicken diversity at high genomic resolution. BMC genomics. 2019;20:345. doi:10.1186/s12864-019-5727-9.

19. Qanbari S. On the Extent of Linkage Disequilibrium in the Genome of Farm Animals. Frontiers in Genetics. 2020;10:1304. doi:10.3389/fgene.2019.01304.

20. VanLiere JM, Rosenberg NA. Mathematical properties of the $r 2$ measure of linkage disequilibrium. Theoretical population biology. 2008;74:130-7.

21. Hinds DA, Kloek AP, Jen M, Chen X, Frazer KA. Common deletions and SNPs are in linkage disequilibrium in the human genome. Nature Genetics. 2006;38:82-5. doi:10.1038/ng1695.

22. Conrad DF, Pinto D, Redon R, Feuk L, Gokcumen O, Zhang Y, et al. Origins and functional impact of copy number variation in the human genome. Nature. 2010;464:704-12. doi:10.1038/nature08516. 
23. Mills RE, Walter K, Stewart C, Handsaker RE, Chen K, Alkan C, et al. Mapping copy number variation by population-scale genome sequencing. Nature. 2011;470:59-65. doi:10.1038/nature09708.

24. Cooper GM, Zerr T, Kidd JM, Eichler EE, Nickerson DA. Systematic assessment of copy number variant detection via genome-wide SNP genotyping. Nature Genetics. 2008;40:1199-203. doi:10.1038/ng.236.

25. Redon R, Ishikawa S, Fitch KR, Feuk L, Perry GH, Andrews TD, et al. Global variation in copy number in the human genome. Nature. 2006;444:444-54. doi:10.1038/nature05329.

26. Kato M, Kawaguchi T, Ishikawa S, Umeda T, Nakamichi R, Shapero MH, et al. Population-genetic nature of copy number variations in the human genome. Hum Mol Genet. 2009;19:761-73. doi:10.1093/hmg/ddp541.

27. McCarroll SA, Kuruvilla FG, Korn JM, Cawley S, Nemesh J, Wysoker A, et al. Integrated detection and population-genetic analysis of SNPs and copy number variation. Nature Genetics. 2008;40:1166-74. doi:10.1038/ng.238.

28. Sudmant PH, Rausch T, Gardner EJ, Handsaker RE, Abyzov A, Huddleston J, et al. An integrated map of structural variation in 2,504 human genomes. Nature. 2015;526:75-81. doi:10.1038/nature15394.

29. Gu W, Zhang F, Lupski JR. Mechanisms for human genomic rearrangements. PathoGenetics. 2008;1:4. doi:10.1186/1755-8417-1-4.

30. Locke DP, Sharp AJ, McCarroll SA, McGrath SD, Newman TL, Cheng Z, et al. Linkage Disequilibrium and Heritability of Copy-Number Polymorphisms within Duplicated Regions of the Human Genome. The American Journal of Human Genetics. 2006;79:275-90. doi:10.1086/505653.

31. Berger S, Schlather M, los Campos G de, Weigend S, Preisinger R, Erbe M, Simianer H. A ScaleCorrected Comparison of Linkage Disequilibrium Levels between Genic and Non-Genic Regions. PLOS ONE. 2015;10:e0141216. doi:10.1371/journal.pone.0141216. 
32. Lee Y-L, Bosse M, Mullaart E, Groenen MAM, Veerkamp RF, Bouwman AC. Functional and population genetic features of copy number variations in two dairy cattle populations. BMC genomics. 2020;21:89. doi:10.1186/s12864-020-6496-1.

33. Xu L, Cole JB, Bickhart DM, Hou Y, Song J, VanRaden PM, et al. Genome wide CNV analysis reveals additional variants associated with milk production traits in Holsteins. BMC genomics. 2014;15:683. doi:10.1186/1471-2164-15-683.

34. Wang L, Xu L, Liu X, Zhang T, Li N, Hay EH, et al. Copy number variation-based genome wide association study reveals additional variants contributing to meat quality in Swine. Scientific Reports. 2015;5:12535. doi:10.1038/srep12535.

35. Wang X, Nahashon S, Feaster TK, Bohannon-Stewart A, Adefope N. An initial map of chromosomal segmental copy number variations in the chicken. BMC genomics. 2010;11:351. doi:10.1186/1471-2164-11-351.

36. Wang Y, Gu X, Feng C, Song C, Hu X, Li N. A genome-wide survey of copy number variation regions in various chicken breeds by array comparative genomic hybridization method. Anim Genet. 2012;43:282-9. doi:10.1111/j.1365-2052.2011.02308.x.

37. Crooijmans RP, Fife MS, Fitzgerald TW, Strickland S, Cheng HH, Kaiser P, et al. Large scale variation in DNA copy number in chicken breeds. BMC genomics. 2013;14:398. doi:10.1186/1471-2164-14398.

38. Tian M, Wang Y, Gu X, Feng C, Fang S, Hu X, Li N. Copy number variants in locally raised Chinese chicken genomes determined using array comparative genomic hybridization. BMC genomics. 2013;14:262. doi:10.1186/1471-2164-14-262.

39. Han R, Yang P, Tian Y, Wang D, Zhang Z, Wang L, et al. Identification and functional characterization of copy number variations in diverse chicken breeds. BMC genomics. 2014;15:934. doi:10.1186/1471-2164-15-934.

40. Jia X, Chen S, Zhou H, Li D, Liu W, Yang N. Copy number variations identified in the chicken using a 60K SNP BeadChip. Anim Genet. 2013;44:276-84. doi:10.1111/age.12009. 
41. Gorla E, Cozzi MC, Román-Ponce SI, Ruiz López FJ, Vega-Murillo VE, Cerolini S, et al. Genomic variability in Mexican chicken population using copy number variants. BMC Genet. 2017;18:61. doi:10.1186/s12863-017-0524-4.

42. Zhang $\mathrm{H}$, Du Z-Q, Dong J-Q, Wang H-X, Shi H-Y, Wang N, et al. Detection of genome-wide copy number variations in two chicken lines divergently selected for abdominal fat content. BMC genomics. 2014;15:517. doi:10.1186/1471-2164-15-517.

43. Rao YS, Li J, Zhang R, Lin XR, Xu JG, Xie L, et al. Copy number variation identification and analysis of the chicken genome using a 60K SNP BeadChip. ps. 2016;95:1750-6. doi:10.3382/ps/pew136.

44. Strillacci MG, Cozzi MC, Gorla E, Mosca F, Schiavini F, Román-Ponce SI, et al. Genomic and genetic variability of six chicken populations using single nucleotide polymorphism and copy number variants as markers. Animal. 2017;11:737-45. doi:10.1017/\$1751731116002135.

45. Lin S, Lin X, Zhang Z, Jiang M, Rao Y, Nie Q, Zhang X. Copy Number Variation in SOX6 Contributes to Chicken Muscle Development. Genes (Basel) 2018. doi:10.3390/genes9010042.

46. Fan W-L, Ng CS, Chen C-F, Lu M-YJ, Chen Y-H, Liu C-J, et al. Genome-wide patterns of genetic variation in two domestic chickens. Genome Biol Evol. 2013;5:1376-92. doi:10.1093/gbe/evt097.

47. Yan Y, Yang N, Cheng HH, Song J, Qu L. Genome-wide identification of copy number variations between two chicken lines that differ in genetic resistance to Marek's disease. BMC genomics. 2015;16:843. doi:10.1186/s12864-015-2080-5.

48. Sohrabi SS, Mohammadabadi M, Wu D-D, Esmailizadeh A. Detection of breed-specific copy number variations in domestic chicken genome. Genome. 2018;61:7-14. doi:10.1139/gen-2017-0016.

49. Seol D, Ko BJ, Kim B, Chai H-H, Lim D, Kim H. Identification of Copy Number Variation in Domestic Chicken Using Whole-Genome Sequencing Reveals Evidence of Selection in the Genome. Animals (Basel) 2019. doi:10.3390/ani9100809.

50. Weng Z, Xu Y, Li W, Chen J, Zhong M, Zhong F, et al. Genomic variations and signatures of selection in Wuhua yellow chicken. PLoS One. 2020;15:e0241137. doi:10.1371/journal.pone.0241137. 
51. Kerstens HHD, Crooijmans RP, Dibbits BW, Vereijken A, Okimoto R, Groenen M am. Structural variation in the chicken genome identified by paired-end next-generation DNA sequencing of reduced representation libraries. BMC genomics. 2011;12:94. doi:10.1186/1471-2164-12-94.

52. Qanbari S, Rubin C-J, Maqbool K, Weigend S, Weigend A, Geibel J, et al. Genetics of adaptation in modern chicken. PLoS Genet. 2019;15:e1007989. doi:10.1371/journal.pgen.1007989.

53. Geibel J, Reimer C, Pook T, Weigend S, Weigend A, Simianer H. How Imputation Can Mitigate Ascertainment Bias; 2021.

54. Belyeu JR, Chowdhury M, Brown J, Pedersen BS, Cormier MJ, Quinlan AR, Layer RM. Samplot: A Platform for Structural Variant Visual Validation and Automated Filtering. bioRxiv 2020. doi:10.1101/2020.09.23.310110.

55. McLaren W, Gil L, Hunt SE, Riat HS, Ritchie GRS, Thormann A, et al. The Ensembl Variant Effect Predictor. Genome Biology. 2016;17:122. doi:10.1186/s13059-016-0974-4.

56. Graffelman J. Exploring Diallelic Genetic Markers: The HardyWeinberg Package. Journal of Statistical Software. 2015;64:1-23.

57. Pedersen BS, Quinlan AR. Duphold: scalable, depth-based annotation and curation of high-confidence structural variant calls. Gigascience. 2019;8:giz040. doi:10.1093/gigascience/giz040.

58. Kranis A, Gheyas AA, Boschiero C, Turner F, Le Yu, Smith S, et al. Development of a high density 600K SNP genotyping array for chicken. BMC genomics. 2013;14:59. doi:10.1186/1471-2164-1459.

59. Groenen MAM, Megens H-J, Zare Y, Warren WC, Hillier LW, Crooijmans RPMA, et al. The development and characterization of a 60K SNP chip for chicken. BMC genomics. 2011;12:274. doi:10.1186/1471-2164-12-274.

60. Liu R, Xing S, Wang J, Zheng M, Cui H, Crooijmans RPMA, et al. A new chicken 55K SNP genotyping array. BMC genomics. 2019;20:410. doi:10.1186/s12864-019-5736-8. 
61. Innovative Management of Animal Genetic Resources (IMAGE). DELIVERABLE D4.5: A standard multi-species chip for genomic assessment of collections. 2020. https://www.imageh2020.eu/deliverable/D4.5_resubmitted_final.pdf. Accessed 17 Aug 2021.

62. Li H. Toward better understanding of artifacts in variant calling from high-coverage samples. Bioinformatics. 2014;30:2843-51. doi:10.1093/bioinformatics/btu356.

63. Bickhart DM, Liu GE. The challenges and importance of structural variation detection in livestock. Frontiers in Genetics. 2014;5:37. doi:10.3389/fgene.2014.00037.

64. Bouwman AC, Derks MF, Broekhuijse ML, Harlizius B, Veerkamp RF. Using short read sequencing to characterise balanced reciprocal translocations in pigs 2020. doi:10.21203/rs.3.rs-28830/v3.

65. Talebi R, Szmatoła T, Mészáros G, Qanbari S. Runs of Homozygosity in Modern Chicken Revealed by Sequence Data. G3 (Bethesda). 2020;10:4615-23. doi:10.1534/g3.120.401860.

66. Qanbari S, Hansen M, Weigend S, Preisinger R, Simianer H. Linkage disequilibrium reveals different demographic history in egg laying chickens. BMC Genet. 2010;11:103. doi:10.1186/1471-2156-11103.

67. Bortoluzzi C, Bosse M, Derks MFL, Crooijmans RPMA, Groenen MAM, Megens H-J. The type of bottleneck matters: Insights into the deleterious variation landscape of small managed populations. Evol Appl. 2020;13:330-41. doi:10.1111/eva.12872.

68. Kyriazis CC, Wayne RK, Lohmueller KE. Strongly deleterious mutations are a primary determinant of extinction risk due to inbreeding depression. Evolution Letters 2020. doi:10.1002/evl3.209.

69. Geibel J, Reimer C, Weigend S, Weigend A, Pook T, Simianer H. How array design creates SNP ascertainment bias. PLoS One. 2021;16:e0245178. doi:10.1371/journal.pone.0245178.

70. Hastings PJ, Lupski JR, Rosenberg SM, Ira G. Mechanisms of change in gene copy number. Nature Reviews Genetics. 2009;10:551-64. doi:10.1038/nrg2593.

71. Meuwissen TH, Hayes BJ, Goddard ME. Prediction of total genetic value using genome-wide dense marker maps. Genetics. 2001;157:1819-29. 
72. Ewels $P$, Magnusson $M$, Lundin S, Käller M. MultiQC: summarize analysis results for multiple tools and samples in a single report. Bioinformatics. 2016;32:3047-8. doi:10.1093/bioinformatics/btw354.

73. Purcell S, Neale B, Todd-Brown K, Thomas L, Ferreira MAR, Bender D, et al. PLINK: a tool set for whole-genome association and population-based linkage analyses. Am. J. Hum. Genet. 2007;81:559-75. doi:10.1086/519795.

74. McKenna A, Hanna M, Banks E, Sivachenko A, Cibulskis K, Kernytsky A, et al. The Genome Analysis Toolkit: A MapReduce framework for analyzing next-generation DNA sequencing data. Genome Res. 2010;20:1297-303. doi:10.1101/gr.107524.110.

75. Li H. A statistical framework for SNP calling, mutation discovery, association mapping and population genetical parameter estimation from sequencing data. Bioinformatics. 2011;27:2987-93. doi:10.1093/bioinformatics/btr509.

76. Chiang C, Layer RM, Faust GG, Lindberg MR, Rose DB, Garrison EP, et al. SpeedSeq: ultra-fast personal genome analysis and interpretation. Nat Methods. 2015;12:966-8. doi:10.1038/nmeth.3505.

77. Jeffares DC, Jolly C, Hoti M, Speed D, Shaw L, Rallis C, et al. Transient structural variations have strong effects on quantitative traits and reproductive isolation in fission yeast. Nature Communications. 2017;8:14061. doi:10.1038/ncomms14061.

78. Pedersen BS, Quinlan AR. Mosdepth: quick coverage calculation for genomes and exomes. Bioinformatics. 2018;34:867-8. doi:10.1093/bioinformatics/btx699.

79. Belyeu JR, Nicholas TJ, Pedersen BS, Sasani TA, Havrilla JM, Kravitz SN, et al. SV-plaudit: A cloudbased framework for manually curating thousands of structural variants. Gigascience 2018. doi:10.1093/gigascience/giy064.

80. Browning BL, Zhou Y, Browning SR. A One-Penny Imputed Genome from Next-Generation Reference Panels. Am. J. Hum. Genet. 2018;103:338-48. doi:10.1016/j.ajhg.2018.07.015. 
81. Pook T, Mayer M, Geibel J, Weigend S, Cavero D, Schoen CC, Simianer H. Improving Imputation Quality in BEAGLE for Crop and Livestock Data. G3 (Bethesda). 2020;10:177-88. doi:10.1534/g3.119.400798.

82. Kent WJ, Sugnet CW, Furey TS, Roskin KM, Pringle TH, Zahler AM, Haussler D. The human genome browser at UCSC. Genome Res. 2002;12:996-1006. doi:10.1101/gr.229102.

83. Köster J, Rahmann S. Snakemake--a scalable bioinformatics workflow engine. Bioinformatics. 2012;28:2520-2. doi:10.1093/bioinformatics/bts480.

751 


\section{Supplementary Files}

This is a list of supplementary files associated with this preprint. Click to download.

- Supplementaryfile1.pdf

- Supplementaryfile2.html

- Supplementaryfile3.html

- Supplementaryfile1.pdf

- Supplementaryfile2.html

- Supplementaryfile4.zip

- Supplementaryfile3.html

- Supplementaryfile4.zip 